\title{
Maxwell's demon-like nonreciprocity by non-Hermitian gyrotropic metasurfaces
}

\author{
Wenyan Wang, ${ }^{1,2}$ Wang Tat Yau $\odot,{ }^{2}$ Yanxia Cui, ${ }^{1}$ Jin Wang $\odot,{ }^{3, *}$ and Kin Hung Fung $\odot^{2, \dagger}$ \\ ${ }^{1}$ College of Physics and Optoelectronics, Key Lab of Advanced Transducers and Intelligent Control System of Ministry of Education, \\ Taiyuan University of Technology, Taiyuan 030024, China \\ ${ }^{2}$ Department of Applied Physics, The Hong Kong Polytechnic University, Hong Kong, China \\ ${ }^{3}$ School of Physics, Southeast University, Nanjing 211189, China
}

(Received 5 October 2020; accepted 26 March 2021; published 19 April 2021)

\begin{abstract}
We show that Maxwell's demon-like nonreciprocity can be supported in a class of non-Hermitian gyrotropic metasurfaces in the linear regime. The proposed metasurface functions as a transmission-only Maxwell's demon operating at a pair of photon energies. Based on multiple scattering theory, we construct a dual-dipole model to explain the underlying mechanism that leads to the antisymmetric nonreciprocal transmission. The results may inspire new designs of compact nonreciprocal devices for photonics.
\end{abstract}

DOI: 10.1103/PhysRevResearch.3.L022006

Lorentz nonreciprocity in electromagnetics refers to unique asymmetrical characteristics in the receivedtransmitted field ratios when the source and detector are exchanged [1,2]. Nonreciprocal elements such as isolators, gyrators, circulators, or directional amplifiers have already become vital components to route signals along desired paths in microwave systems [3-8]. Photonic isolation for protecting active components from backward scattering is a key requirement in stable laser devices and integrated photonic circuits [9-13]. The standard approach to break Lorentz reciprocity in the linear regime relies on the magneto-optical (MO) effect, i.e., the use of MO materials with an asymmetrical permeability (or permittivity) tensor [14-17], or parametric time-modulation composites to completely reproduce the MO effect [9,18-22]. An alternative strategy based on nonlinear materials has also been proposed [23-30]. There are respective benefits as well as limitations in different approaches [31,32]. It is believed that metamaterials or metasurfaces may offer unprecedented avenues to overcome some of the limitations.

For free-space photonics, linear elements in the form of Lorentz nonreciprocal metasurfaces are experiencing a strong surge of interest owing to their ultrathin thickness, conceptual simplicity, and potential conformability. Photonics could be a promising experimental platform for investigating the role of information in the statistical mechanics associated with Maxwell's demon [33], which is a nonreciprocal device controlling the passage of particles through a thin wall according to their energy. To date, metasur-

\footnotetext{
*jinwang@seu.edu.cn

${ }^{\dagger}$ khfung@polyu.edu.hk

Published by the American Physical Society under the terms of the Creative Commons Attribution 4.0 International license. Further distribution of this work must maintain attribution to the author(s) and the published article's title, journal citation, and DOI.
}

faces supporting the Maxwell's demon-like selection of photons have not yet been addressed. In this Letter, we propose a non-Hermitian gyrotropic metasurface composed of dimer unit cells to achieve such demon-like antisymmetric transmittance spectra for two photon energies at normal incidence. It should be noted that there were successful engineering designs supporting nonreciprocal absorption or reflection using gyromagnetic cylinders [34-36], but none of the mentioned designs could support demon-like action for selective transmittance. Figure 1 schematically illustrates the Maxwell's demon-like nonreciprocity operation at two photon energies, where the demon controls nearly perfect one-way penetration of two different energy photons on a particular side of the metasurface and, meanwhile, nearly complete rejection of reverse flow. The underlying mechanism of such demon-like nonreciprocity is explicitly revealed by the cooperative effects of magnetic rotating dipole resonance in gyromagnetic cylinders and electric dipole response in dielectric cylinders in the proposed structure. However, non-Hermiticity is found to be essential to the isolation properties, corresponding to the energy consumption of Maxwell's demon.

Let us consider a metasurface as shown in Figs. 2(a) and 2(b), which is composed of an array of gyromagnetic and isotropic dielectric cylinders arranged in the $x$ direction with a lattice constant $\Lambda$. For the sake of simplicity, the gyrotropic and dielectric cylinders have the same radius $r$, and the centerto-center distance between the adjacent cylinders is denoted by $a$. A small lattice constant is chosen such that there are no diffracted propagating beams for the metasurface over the frequency range of interest. Under an external static magnetic field $H_{0}$ along the $-z$ axis, the chosen gyromagnetic material, i.e., yttrium iron garnet (YIG), is described by a permittivity $\epsilon_{m}$ and an asymmetric permeability tensor $\tilde{\mu}$ [37]:

$$
\tilde{\mu}=\mu_{0}\left(\begin{array}{ccc}
\mu_{1} & i \mu_{2} & 0 \\
-i \mu_{2} & \mu_{1} & 0 \\
0 & 0 & 1
\end{array}\right),
$$


(a)

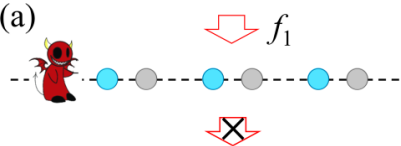

(b)

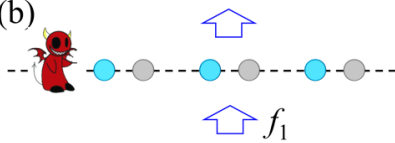

(c)

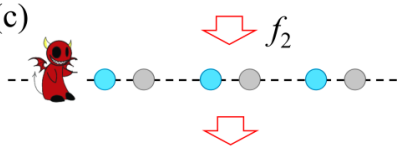

(d)

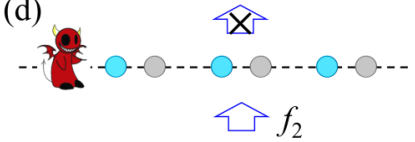

FIG. 1. Schematic diagram of antisymmetric Maxwell's demonlike operation supported by a metasurface composed of dimer unit cells. The metasurface (a) forbids the penetration of photons with frequency $f_{1}$ in downward incidence, while it (b) allows the penetration of photons with frequency $f_{1}$ in upward incidence. The same metasurface also (c) allows the penetration of photons with frequency $f_{2}$ in downward incidence, while it (d) forbids the penetration of photons with frequency $f_{2}$ in upward incidence.

with the elements $\mu_{1}=1+\omega_{m} \omega_{0} /\left(\omega_{0}^{2}-\omega^{2}\right)$ and $\mu_{2}=$ $\omega_{m} \omega /\left(\omega_{0}^{2}-\omega^{2}\right)$, where $\omega_{0}=\gamma H_{0}$ is the precession frequency, $\omega_{m}=\gamma 4 \pi M_{s}, 4 \pi M_{s}$ is the saturation magnetization, $\gamma$ is the gyromagnetic ratio, $\mu_{0}$ is the vacuum permeability, and $\omega$ is the angular frequency. The complex index of refraction of the isotropic dielectric cylinder is $n_{d}$, where the imaginary part is denoted as $k$. This imaginary part contributes to energy loss, which makes the dimer system non-Hermitian, resulting in nonreciprocal transmittance based on asymmetric absorption. Here, we limit the case to transverse electric (TE) polarized waves (electric field along the $z$ direction), and the
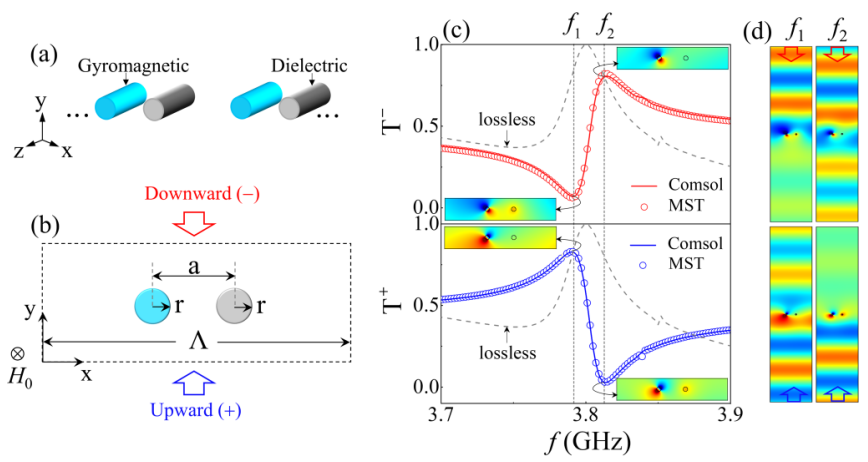

FIG. 2. (a) Schematic diagram of the metasurface consisting of gyromagnetic (blue) and isotropic dielectric (gray) cylinders. (b) Top view of the unit cell of the periodic system in (a). Periodic conditions are applied on the $x$ axis throughout all calculations. Plane waves illuminating from $-y$ and $+y$ directions are denoted as downward incidence by a red arrow and upward incidence by a blue arrow, respectively. (c) The numerical and analytical transmittance spectra, represented as solid lines and open circles, respectively, under downward (panel at top) and upward (panel at bottom) incidences. The transmittance spectra of a lossless system in both directions are shown as black dashed lines. The insets show the $\mathbf{E}_{z}$ field patterns of those cylinders at those transmittance extrema. (d) The transient electric field $\mathbf{E}_{z}$ at $f_{1}$ and $f_{2}$. The marked arrows indicate the direction of the incident waves. Material parameters are $\epsilon_{m}=15, H_{0}=500$ Oe, and $M_{s}=1750 /(4 \pi) \mathrm{G}$, and $n_{d}=10+2.8 i$. Geometrical parameters are $\Lambda=50 \mathrm{~mm}, r=1 \mathrm{~mm}$, and $a=12 \mathrm{~mm}$. $e^{-i \omega t}$ time-dependent convention for the harmonic field is used throughout this Letter.

We start with a scattering problem under the illumination of normal incident plane waves with the wave vector along the downward $(-y)$ direction and the upward $(+y)$ direction, as shown in Fig. 2(b). Previously, nonreciprocal absorption or reflection phenomena at oblique incidence have been demonstrated in microstructures consisting of arrays of single gyromagnetic cylinders [34-36]. To support photonic isolation at normal incidence for Maxwell's demon-like action, additional symmetries (such as $\pi$-rotation symmetry about the $z$ axis) must be broken (which is provided by the addition of dielectric cylinders). Under the assumption of the point-dipole approximation, we may create a dual-dipole field model for the entire metasurface system by treating the gyromagnetic cylinder and the dielectric cylinder as a rotating magnetic dipole (MD) with angular quantum number -1 and damping electric dipole (ED) with angular momentum quantum number 0 , respectively, in multiple scattering theory (MST) [38]. In such a dual-dipole approximation, a $2 \times 2$ matrix problem can be formulated, and the scattering fields of the cylinders are related to the incident waves by

$$
\left(\begin{array}{c}
s_{-1}^{Y} \\
s_{0}^{d}
\end{array}\right)^{\mp}=\hat{W}^{-1}\left(\begin{array}{c}
\mp 1 \\
1
\end{array}\right),
$$

where $s_{-1}^{Y}$ and $s_{0}^{d}$ represent the scattering fields of the gyromagnetic and dielectric cylinders, respectively, and $\hat{W}^{-1}$ is an invertible response matrix in the eigenresponse theory $[38,39]$. The above equation with a sign of "+" and "-" represents the upward and downward incidence, respectively. The transmittance at normal incidence is then given by [38]

$$
\mathrm{T}^{\mp}=\left|\frac{\left(L-\frac{2}{k_{0} \Lambda}+\frac{1}{b_{0}^{d}}\right)\left(L-\frac{2}{k_{0} \Lambda}+\frac{1}{b_{-1}^{Y}}\right)-\left(\frac{2}{k_{0} \Lambda} \pm \xi\right)^{2}}{\left(L+\frac{1}{b_{0}^{d}}\right)\left(L+\frac{1}{b_{-1}^{Y}}\right)-\xi^{2}}\right|^{2}
$$

where $b$ is the Mie scattering coefficient [40,41] and $L$ and $\xi$ are the lattice sum and the relative lattice sum $[42,43]$ signifying the contribution of the intraspecific and interspecific coupling, respectively. The superscripts $Y$ and $d$ following the Mie coefficient $b$ represent the gyromagnetic and dielectric material, respectively, and the subscripts $(-1$ and 0$)$ behind the symbols denote the angular momentum order [38]. The nonreciprocity is triggered by the cross term $\pm 4 \xi / k_{0} \Lambda$ due to the interference of the lattice coupling $2 / k_{0} \Lambda$ and purely imaginary interspecific coupling $\xi$ [38]. It should be noted that removal of absorption in the dielectric cylinder (i.e., $k=0$ ) extinguishes the nonreciprocal transmittance but the phase of transmission coefficients remains different.

The closed form in Eq. (3) directly illustrates the antisymmetric nonreciprocal transmittance phenomena, as indicated in Fig. 2(c) with scatter points shown as open circles. $\mathrm{Nu}$ merical results calculated with COMSOL MULTIPHYSICS are also shown in Fig. 2(c) as solid lines, which shows a strong agreement with the analytical results. The transient $\mathbf{E}_{z}$ field patterns of two tremendous transmittance difference points at frequency $f_{1}=3.79 \mathrm{GHz}$ [Fig. 2(d), left panel] and $f_{2}=$ $3.814 \mathrm{GHz}$ [Fig. 2(d), right panel], where the local minimum 
of the spectrum in one direction nearly corresponds to the local maximum of the spectrum in the opposite direction, demonstrate an imperfect Maxwell's demon function similar to Fig. 1. At both $f_{1}$ and $f_{2}$, the electric field strength of the dielectric cylinder in low transmittance cases [Fig. 2(c), Insets, right circle] is relatively higher than that in high transmittance cases. This electric field strength may indicate the system absorption and the nonreciprocal transmittance because the loss is only introduced in the dielectric cylinder. Therefore, by removing the loss in the dielectric cylinder, the transmittance spectra become identical for both directions [Fig. 2(c), black dashed lines]. The Maxwell's demon in the dimer metasurface therefore seems to be conceptually similar to the statistical Maxwell's demon in the sense of the energy consumption for nonreciprocal flow. However, the demon-like action is imperfect in this system (e.g., it does not completely forbid or allow the penetration of photons on both sides [44]).

For two-port nonreciprocal systems, absorption loss is a necessary condition to attain nonreciprocal transmittance [1]. The implement of intrinsic loss in real systems, however, affects the nonreciprocal performance in a complex way. In many nonreciprocal designs, intrinsic loss leads to the inevitable insert loss of devices for attaining ideal nonreciprocity $[12,45,46]$, while the two-port magnon-photon cavity system has demonstrated the isolation in the presence of damping of modes [7]. In our case, the metasurface effectively manipulates the nonreciprocity by simply controlling the absorption of dielectric cylinders. Such a simple phenomenon is based on the same reflectance on both sides of the metasurface [38] and the conservation of energy. Therefore $\Delta \mathrm{T}=\mathrm{T}^{-}-\mathrm{T}^{+}=-\Delta \mathrm{A}$, where $\Delta \mathrm{A}=\mathrm{A}^{-}-\mathrm{A}^{+}$is the difference in absorption.

$$
\mathrm{A}^{\mp}=\frac{4}{k_{0} \Lambda} \beta\left|\left(s_{0}^{d}\right)^{\mp}\right|^{2}
$$

denotes the absorption in each incident direction, and $\beta=$ $\operatorname{Re}\left(L+1 / b_{0}^{d}-2 / k_{0} \Lambda\right)$ represents the damping coefficient.

In Fig. 3, we show the relations among the transmittance (T) from Eq. (3), the absorption (A) in Eq. (4), and the scattered field amplitude $\left(s_{0}^{d}\right)$ of the dielectric cylinder. It is found that all three quantities show antisymmetric features with two opposite extrema located at $f=f_{1}$ and $f=f_{2}$, sandwiching the single-cylinder gyromagnetic resonance at $f=f_{0}=3.80 \mathrm{GHz}$. The asymmetric absorption characteristics, following the dependence of $\left|\left(s_{0}^{d}\right)^{\mp}\right|^{2}$, should therefore be the reason of triggering the nonreciprocal transmittance in the presence of $k$ (i.e., the imaginary part of the dielectric constant). As $k$ increases, the transmittance difference $(\Delta \mathrm{T})[$ Fig. 4(a), density plot] and absorption difference $(\Delta \mathrm{A})$ [Fig. 4(b), density plot] become different in sign around $f_{1}$ and $f_{2}$. The extrema of $\Delta \mathrm{A}$ by Eq. (4) (Fig. 4, white circles and black spheres) also appear at $f=f_{1}$ and $f=f_{2}$. The locations of those extrema near $f_{1}$ and $f_{2}$ are insensitive to $k$ since they are nearly pinned by the single-cylinder gyromagnetic resonance, corresponding to the frequency which satisfies $\operatorname{Im}\left(1 / b_{-1}^{Y}+L\right)=0$. The $k$ dependences of the numerical and analytical values of $\Delta \mathrm{A}$ at the two frequencies show an excellent agreement [see Fig. 4(c)]. Therefore the scattered field amplitude of the dielectric cylinder is a good quantity to illustrate the nonreciprocity mechanism.

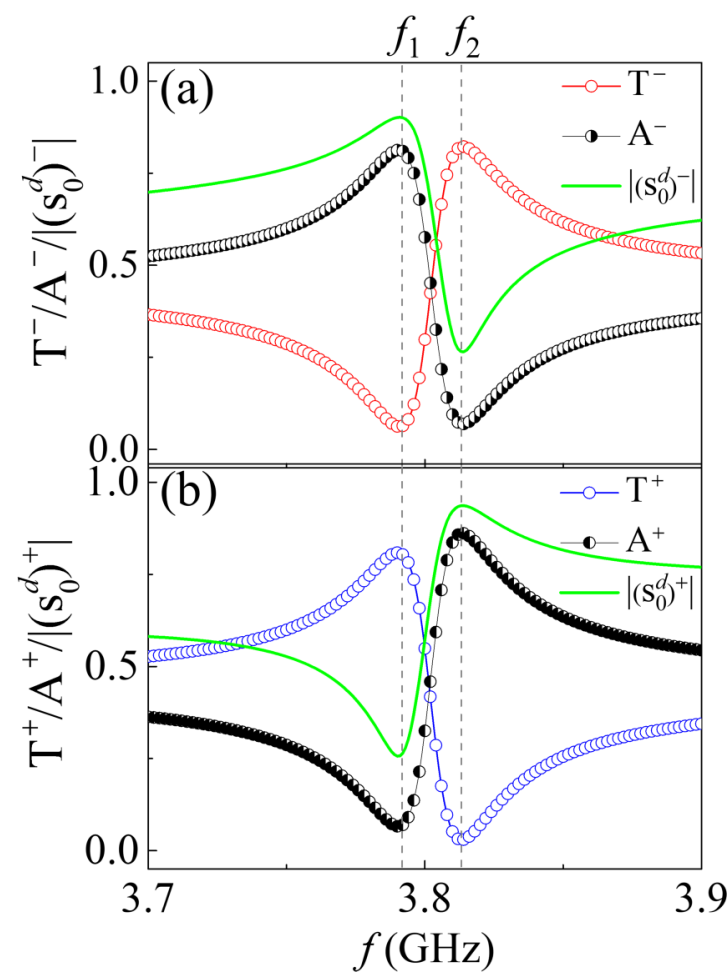

FIG. 3. Comparison of analytical transmittance (T) spectra (solid lines with open circles), analytical absorption (A) spectra (solid lines with half-filled circles) from Eq. (4), and the magnitude of the scattered field of the dielectric cylinder $\left(\left|s_{0}^{d}\right|\right)$ (green solid lines) under (a) downward incidence and (b) upward incidence, denoted by the superscripts - and + , respectively. The local extrema of the three spectra under two incidences are simultaneously located at $f_{1}$ and $f_{2}$, marked by the black dashed lines.

To gain a deeper insight into the cooperative effect of MD in the gyromagnetic cylinder and ED in the dielectric one, we firstly schematically illustrate the cooperative effect in the dimer metasurface. The isolated gyromagnetic cylinder array [Fig. 5(a), blue cylinder] and the isolated dielectric cylinder array [Fig. 5(a), gray cylinder] exist in their own collective resonance modes. By merging these two arrays, these two collective modes will couple and form two hybrid modes [Fig. 5(a)]. In the eigenresponse theory [39], the eigenvalues of the response matrix $\hat{W}^{-1}$ are given by $[38,47]$

$$
1 / w_{\chi}=-\frac{\frac{1}{b_{0}^{d}}+\frac{1}{b_{-1}^{Y}}+2 L+(-1)^{\chi-1} \sqrt{\left(\frac{1}{b_{0}^{d}}-\frac{1}{b_{-1}^{Y}}\right)^{2}+4 \xi^{2}}}{2\left[\left(L+\frac{1}{b_{0}^{d}}\right)\left(L+\frac{1}{b_{-1}^{Y}}\right)-\xi^{2}\right]}
$$

for the corresponding eigenvector $\mathbf{g}_{x}$, where

$$
\mathbf{g}_{\chi}=\left(\begin{array}{l}
\varrho^{Y} \\
\varrho^{d}
\end{array}\right)_{\chi}
$$

represents the $\chi$ th eigenmode of the dimer system. $\varrho^{Y}$ and $\varrho^{d}$ in Eq. (6) denote the components corresponding to the gyromagnetic and dielectric cylinders, respectively, in the unit cell after cooperative coupling. 

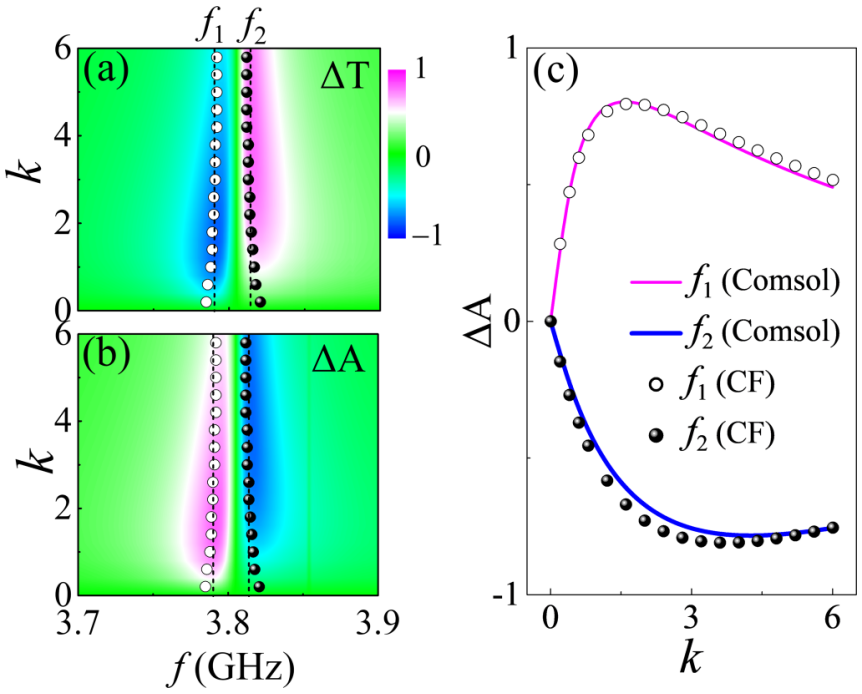

FIG. 4. The effects of $k$, the imaginary part of the dielectric constant, on (a) numerical transmission difference $\Delta \mathrm{T}$ and (b) numerical absorption difference $\Delta \mathrm{A}$. The white circles and black spheres in (a) and (b) represent the peak and depth locations of difference of A in Eq. (4), respectively. (c) The vertical cross section of (b) at $f_{1}$ and $f_{2}$, showing the effect of $k$ on $\Delta \mathrm{A}$ at those frequencies. Excellent agreements are obtained between the closed form (CF) in Eq. (4) and numerical results.

The normalization of eigenmodes and the biorthogonality relation suggests [38]

$$
\left(\begin{array}{l}
\varrho^{Y} \\
\varrho^{d}
\end{array}\right)_{2}=\left(\begin{array}{c}
-\varrho^{d} \\
\varrho^{Y}
\end{array}\right)_{1},
$$

which quantitatively classifies $\mathbf{g}^{\mathrm{T}}$ and $\mathbf{g}^{\mathrm{A}}$ as $\mathbf{g}^{\mathrm{T}}:\left|\varrho^{Y}\right|>\left|\varrho^{d}\right|$ and $\mathbf{g}^{\mathrm{A}}:\left|\varrho^{Y}\right| \leqslant\left|\varrho^{d}\right|$. Following the understanding of the absorption and scattered field amplitude of the dielectric cylinder, $\mathbf{g}^{\mathrm{A}}$ and $\mathbf{g}^{\mathrm{T}}$ can be realized as the loss dominant mode and transmissive dominant mode, respectively. $\left|\varrho^{d}\right|$ in the two eigenmodes shows the antisymmetric pairs of step-functionlike characteristics with the transition point at $f_{0} \simeq 3.80 \mathrm{GHz}$ [Fig. 5(b)]. This result indicates that $\mathbf{g}_{1}$ belongs to $\mathbf{g}^{\mathrm{A}}$ before $f_{0}$ and $\mathbf{g}_{2}$ belongs to $\mathbf{g}^{\mathrm{A}}$ after $f_{0}$. As $\left|\varrho^{d}\right|$ in the two eigenmodes shows a large difference before or after $f_{0}$, the absorption seems to be dominated by $\mathbf{g}^{\mathbf{A}}$ only, and these two eigenmodes would likely be the on-off state of the gate controlled by the Maxwell's demon.

To illustrate whether the gate would be opened or closed by the Maxwell's demon, we write the scattered field of the cylinders through eigendecomposition as

$$
\left(\begin{array}{c}
s_{-1}^{Y} \\
s_{0}^{d}
\end{array}\right)^{\mp}=\sum_{\chi=1}^{2} \eta_{\chi}^{\mp} \mathbf{g}_{\chi},
$$

where $\eta_{\chi}^{\mp}=\left(\varrho_{\chi}^{d} \mp \varrho_{\chi}^{Y}\right) / w_{\chi}$ is the complex excitation coefficient of the $\chi$ th eigenmode, sensitively dependent on the downward (-) or upward (+) illumination. Equation (8) shows the contribution of eigenmodes to the scattered field amplitude according to their excitation coefficients. As the absorption should be mainly contributed by the loss dominant eigenmode, a discussion of that excitation coefficient would
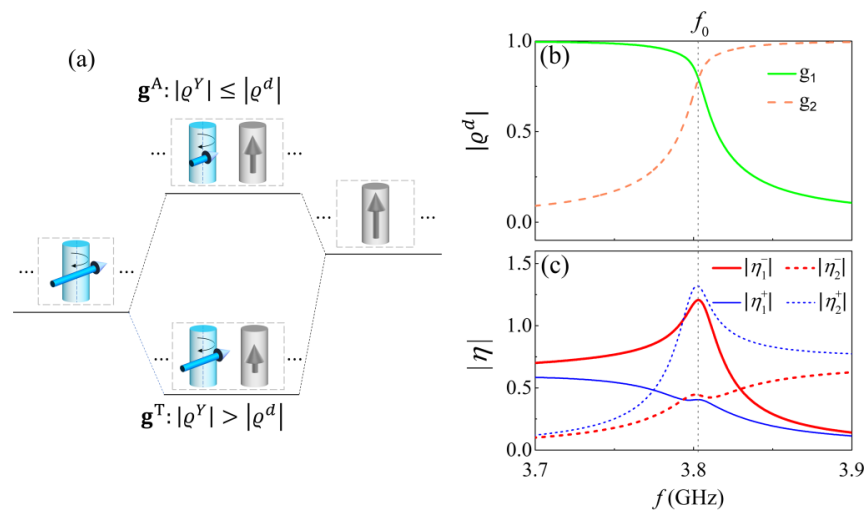

FIG. 5. (a) Schematic diagram of mode hybridization of a gyromagnetic (blue) and dielectric (gray) cylinder array. The collective resonance modes of an isolated gyromagnetic cylinder array (MD, denoted as a blue arrow) and an isolated dielectric cylinder array (ED, denoted as a gray arrow) are hybridized into absorptive dominant modes $\mathbf{g}^{\mathrm{A}}\left(\left|\varrho^{Y}\right| \leqslant\left|\varrho^{d}\right|\right)$ and transmissive dominant modes $\mathbf{g}^{\mathrm{T}}$ $\left(\left|\varrho^{Y}\right|>\left|\varrho^{d}\right|\right)$ by merging the two arrays. (b) The magnitude of the component corresponding to the dielectric cylinder $\left|\varrho^{d}\right|$ in two eigenvectors, $\mathbf{g}_{1}$ (green line) and $\mathbf{g}_{2}$ (orange dashed line). The crossing point of the two lines is found at $f_{0}=3.80 \mathrm{GHz}$. (c) The magnitude of the excitation coefficient, $\left|\eta_{1,2}^{ \pm}\right|$, to the two eigenvectors, $\mathbf{g}_{1}$ and $\mathbf{g}_{2}$, under upward (or downward) wave excitation. The subscripts 1, 2 of $|\eta|$ refer to the excitation of the eigenmodes with corresponding indices. The superscripts - and + denote the downward and upward incidences, respectively.

illustrate the absorption of the system, which would be interpreted as the probability that Maxwell's demon would close the gate.

Before the frequency $f_{0}$, where $\mathbf{g}_{1}$ is $\mathbf{g}^{\mathrm{A}},\left|\eta_{1}^{-}\right|$gradually increases to a maximum [Fig. 5(c), red solid line], whereas $\left|\eta_{1}^{+}\right|$drops to a local minimum [Fig. 5(c), blue solid line], giving rise to the asymmetrical absorption: the rise in the downward absorption and gradual drop in the upward absorption. Similarly, after $f_{0}$, where $\mathbf{g}_{2}$ is $\mathbf{g}^{\mathrm{A}}$, the distinct variation of excitation coefficients of $\left|\eta_{2}^{-}\right|$[Fig. 5(c), red dashed line] and $\left|\eta_{2}^{+}\right|$[Fig. 5(c), blue dashed line] can produce the remarkable nonreciprocity in absorption, subject to the light illumination. As a consequence, it is revealed that the excitation of the dominant absorptive modes governs the nonreciprocal transmission and thereby the intriguing demon-like operation. It is worth noting that the dimer system remains nonreciprocal by following similar arguments, when the intrinsic material absorption is only introduced in the gyrotropic cylinder.

In summary, we propose an ultrathin metasurface that can produce antisymmetric nonreciprocal transmission peakand-depth pairs. The metasurface functions as an imperfect Maxwell's demon for normal incident photons. It is found that the antisymmetric (nonreciprocal) transmittance is induced directly by the asymmetrical absorption in lossy dielectric cylinders under the cooperative effect of the rotating magnetic dipole and linear electric dipole excited in the metasurface. Eigenresponse theory using a simple $2 \times 2$ matrix truncated from multiple scattering theory is employed to reveal the underlying mechanism. Moreover, 
the ultrathin property of the proposed metasurface is easier to realize in practical applications compared with the traditional bulky Lorentz nonreciprocal devices. Our results may inspire designs of novel nonreciprocal devices for photonics.

This work was supported by the Hong Kong Research Grants Council (Grants No. AoE/P-02/12, No. C6013-18G, and No. 15301917). W.W. and Y.C. acknowledge the sup- port from the National Natural Science Foundation of China (Grants No. 61905173 and No. 61775156) and the Natural Science Foundation of Shanxi Province (Grants No. 201701D211002 and No. 201801D221029). J.W. acknowledges the support from the Natural Science Foundation of Jiangsu Province (Grant No. BK20181263). We thank Kai Fung Lee, Wai Chun Wong, and C. T. Chan for fruitful discussions.

W.T.Y. and W.W. contributed equally to this work.
[1] C. Caloz, A. Alù, S. Tretyakov, D. Sounas, K. Achouri, and Z.-L. Deck-Léger, Electromagnetic Nonreciprocity, Phys. Rev. Appl. 10, 047001 (2018).

[2] S. R. K. Rodriguez, V. Goblot, N. C. Zambon, A. Amo, and J. Bloch, Nonreciprocity and zero reflection in nonlinear cavities with tailored loss, Phys. Rev. A 99, 013851 (2019).

[3] N. R. Bernier, L. D. Tóth, A. Koottandavida, M. A. Ioannou, D. Malz, A. Nunnenkamp, A. Feofanov, and T. Kippenberg, Nonreciprocal reconfigurable microwave optomechanical circuit, Nat. Commun. 8, 604 (2017).

[4] G. A. Peterson, F. Lecocq, K. Cicak, R. W. Simmonds, J. Aumentado, and J. D. Teufel, Demonstration of Efficient Nonreciprocity in a Microwave Optomechanical Circuit, Phys. Rev. X 7, 031001 (2017).

[5] Z. Wang, Y. Chong, J. D. Joannopoulos, and M. Soljačić, Observation of unidirectional backscattering-immune topological electromagnetic states, Nature (London) 461, 772 (2009).

[6] F. Lecocq, L. Ranzani, G. A. Peterson, K. Cicak, R. W. Simmonds, J. D. Teufel, and J. Aumentado, Nonreciprocal Microwave Signal Processing with a Field-Programmable Josephson Amplifier, Phys. Rev. Appl. 7, 024028 (2017).

[7] Y.-P. Wang, J. W. Rao, Y. Yang, P.-C. Xu, Y. S. Gui, B. M. Yao, J. Q. You, and C.-M. Hu, Nonreciprocity and Unidirectional Invisibility in Cavity Magnonics, Phys. Rev. Lett. 123, 127202 (2019).

[8] K. M. Sliwa, M. Hatridge, A. Narla, S. Shankar, L. Frunzio, R. J. Schoelkopf, and M. H. Devoret, Reconfigurable Josephson Circulator/Directional Amplifier, Phys. Rev. X 5, 041020 (2015).

[9] D. L. Sounas and A. Alù, Non-reciprocal photonics based on time modulation, Nat. Photonics 11, 774 (2017).

[10] Z. Shen, Y.-L. Zhang, Y. Chen, C.-L. Zou, Y.-F. Xiao, X.-B. Zou, F.-W. Sun, G.-C. Guo, and C.-H. Dong, Experimental realization of optomechanically induced non-reciprocity, Nat. Photonics 10, 657 (2016).

[11] Z. Yu and S. Fan, Complete optical isolation created by indirect interband photonic transitions, Nat. Photonics 3, 91 (2009).

[12] A. B. Khanikaev, S. H. Mousavi, G. Shvets, and Y. S. Kivshar, One-Way Extraordinary Optical Transmission and Nonreciprocal Spoof Plasmons, Phys. Rev. Lett. 105, 126804 (2010).

[13] L. Bi, J. Hu, P. Jiang, D. H. Kim, G. F. Dionne, L. C. Kimerling, and C. Ross, On-chip optical isolation in monolithically integrated non-reciprocal optical resonators, Nat. Photonics 5, 758 (2011).

[14] M. Shalaby, M. Peccianti, Y. Ozturk, and R. Morandotti, A magnetic non-reciprocal isolator for broadband terahertz operation, Nat. Commun. 4, 1558 (2013).
[15] W. C. Wong, W. Wang, W. T. Yau, and K. H. Fung, Topological theory for perfect metasurface isolators, Phys. Rev. B 101, 121405(R) (2020).

[16] J. Wang, H. Y. Dong, Q. Y. Shi, W. Wang, and K. H. Fung, Coalescence of nonreciprocal exceptional points in magnetized PT-symmetric systems, Phys. Rev. B 97, 014428 (2018).

[17] Q. Y. Shi, H. Y. Dong, K. H. Fung, Z.-g. Dong, and J. Wang, Optical non-reciprocity induced by asymmetrical dispersion of Tamm plasmon polaritons in terahertz magnetoplasmonic crystals, Opt. Express 26, 33613 (2018).

[18] H. Lira, Z. Yu, S. Fan, and M. Lipson, Electrically Driven Nonreciprocity Induced by Interband Photonic Transition on a Silicon Chip, Phys. Rev. Lett. 109, 033901 (2012).

[19] D. L. Sounas, C. Caloz, and A. Alu, Giant non-reciprocity at the subwavelength scale using angular momentum-biased metamaterials, Nat. Commun. 4, 2407 (2013).

[20] N. A. Estep, D. L. Sounas, J. Soric, and A. Alù, Magneticfree non-reciprocity and isolation based on parametrically modulated coupled-resonator loops, Nat. Phys. 10, 923 (2014).

[21] K. Fang, Z. Yu, and S. Fan, Photonic Aharonov-Bohm Effect Based on Dynamic Modulation, Phys. Rev. Lett. 108, 153901 (2012).

[22] K. Fang, Z. Yu, and S. Fan, Realizing effective magnetic field for photons by controlling the phase of dynamic modulation, Nat. Photonics 6, 782 (2012).

[23] H. Ramezani, T. Kottos, R. El-Ganainy, and D. N Christodoulides, Unidirectional nonlinear $P T$-symmetric optical structures, Phys. Rev. A 82, 043803 (2010).

[24] T. Espinosa-Ortega, T. C. H. Liew, and I. A. Shelykh, Optical diode based on exciton-polaritons, Appl. Phys. Lett. 103, 191110 (2013).

[25] L. Chang, X. Jiang, S. Hua, C. Yang, J. Wen, L. Jiang, G. Li, G. Wang, and M. Xiao, Parity-time symmetry and variable optical isolation in active-passive-coupled microresonators, Nat. Photonics 8, 524 (2014).

[26] B. Peng, Ş. K. Özdemir, F. Lei, F. Monifi, M. Gianfreda, G. L. Long, S. Fan, F. Nori, C. M. Bender, and L. Yang, Parity-timesymmetric whispering-gallery microcavities, Nat. Phys. 10, 394 (2014).

[27] Y. Kominis, T. Bountis, and S. Flach, The asymmetric active coupler: Stable nonlinear supermodes and directed transport, Sci. Rep. 6, 33699 (2016).

[28] D. L. Sounas and A. Alù, Time-Reversal Symmetry Bounds on the Electromagnetic Response of Asymmetric Structures, Phys. Rev. Lett. 118, 154302 (2017). 
[29] D. L. Sounas, J. Soric, and A. Alu, Broadband passive isolators based on coupled nonlinear resonances, Nat. Electron. 1, 113 (2018).

[30] D. Ballarini, M. De Giorgi, E. Cancellieri, R. Houdré, E. Giacobino, R. Cingolani, A. Bramati, G. Gigli, and D. Sanvitto, All-optical polariton transistor, Nat. Commun. 4, 1778 (2013).

[31] Y. Shi, Z. Yu, and S. Fan, Limitations of nonlinear optical isolators due to dynamic reciprocity, Nat. Photonics 9, 388 (2015).

[32] S. A. Mann, D. L. Sounas, and A. Alù, Nonreciprocal cavities and the time-bandwidth limit, Optica 6, 104 (2019).

[33] M. D. Vidrighin, O. Dahlsten, M. Barbieri, M. S. Kim, V. Vedral, and I. A. Walmsley, Photonic Maxwell's Demon, Phys. Rev. Lett. 116, 050401 (2016).

[34] S. Chui, S. Liu, and Z. Lin, Reflected wave of finite circulation from magnetic photonic crystals, J. Phys.: Condens. Matter 22, 182201 (2010).

[35] J. Yu, H. Chen, Y. Wu, and S. Liu, Magnetically manipulable perfect unidirectional absorber based on nonreciprocal magnetic surface plasmon, EPL 100, 47007 (2012).

[36] C. Ju, R.-X. Wu, Z. Li, Y. Poo, S.-Y. Liu, and Z.-F. Lin, Manipulating electromagnetic wave propagating non-reciprocally by a chain of ferrite rods, Opt. Express 25, 22096 (2017).

[37] J. Jin, S. Liu, Z. Lin, and S. T. Chui, Effective-medium theory for anisotropic magnetic metamaterials, Phys. Rev. B 80, 115101 (2009).

[38] See Supplemental Material at http://link.aps.org/supplemental/ 10.1103/PhysRevResearch.3.L022006 for the derivation of the dual-dipole model from multiple scattering theory and spectral analysis.
[39] K. H. Fung, A. Kumar, and N. X. Fang, Electron-photon scattering mediated by localized plasmons: A quantitative analysis by eigen-response theory, Phys. Rev. B 89, 045408 (2014).

[40] C. F. Bohren and D. R. Huffman, Absorption and Scattering of Light by Small Particles (John Wiley, New York, 2008).

[41] J. Wang, K. F. Lee, H. Y. Dong, Z.-G. Dong, S. F. Yu, and K. H. Fung, Collective resonances in a circular array of gyromagnetic rods, Phys. Rev. B 101, 045425 (2020).

[42] K. Yasumoto, Electromagnetic Theory and Applications for Photonic Crystals (CRC, Boca Raton, 2005).

[43] L. C. Botten, N.-A. P. Nicorovici, A. A. Asatryan, R. C. McPhedran, C. M. de Sterke, and P. A. Robinson, Formulation for electromagnetic scattering and propagation through grating stacks of metallic and dielectric cylinders for photonic crystal calculations. Part I. Method, J. Opt. Soc. Am. A 17, 2165 (2000).

[44] See Fig. S1(a) of the Supplemental Material [38].

[45] H. Y. Dong, J. Wang, and T. J. Cui, One-way Tamm plasmon polaritons at the interface between magnetophotonic crystals and conducting metal oxides, Phys. Rev. B 87, 045406 (2013).

[46] H. Y. Dong, J. Wang, and K. H. Fung, One-way optical tunneling induced by nonreciprocal dispersion of Tamm states in magnetophotonic crystals, Opt. Lett. 38, 5232 (2013).

[47] E. N. Bulgakov and A. F. Sadreev, Bloch bound states in the radiation continuum in a periodic array of dielectric rods, Phys. Rev. A 90, 053801 (2014). 